\section{Will women soon outrun men?}

SIR - The mean running velocity $(\bar{V})$ is a crucial determinant of the metabolic demands imposed by competitive running events ${ }^{1,2}$. Its historical progression, therefore, is likely to be important in understanding the physiological determinants of the seemingly inexorable progression of record performances.

We therefore established $\bar{V}$ as a function of historical time $(t)$ for the world records at all the standard Olympic events from the $200 \mathrm{~m}$ to the marathon
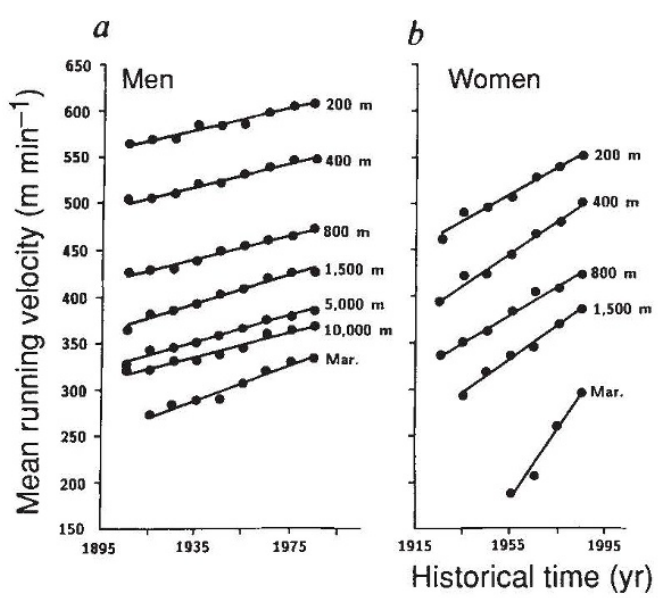

World record progression, expressed as mean running velocity versus historical time, for men (a) and women (b), with best-fit linear regressions (solid lines) superimposed. In $c$, the regression lines for the common events for men and women (solid lines) are extrapolated (dashed lines) to their points of intersection; the predicted world record times at these intersection points are shown in parentheses (h:min:s)

$(42,195 \mathrm{~m})$ for men, decade-by-decade, throughout this century ${ }^{3,4}$. We were able to establish this relationship only for events up to $1,500 \mathrm{~m}$ since the 1920 s for women; data were inadequate for the 5,000 and $10,000 \mathrm{~m}$, although we judged there to be sufficient for the marathon.

In men, the progression of $\bar{V}$ appears to be a linear function of $t$, with slopes for the different events being remarkably similar ( $a$ in the figure) - in agreement with the results of Ryder et al. ${ }^{5}$. These ranged from 5.69 to $7.57 \mathrm{~m} \mathrm{~min}^{-1}$ decade, with no systematic variation with increasing race distance. The marathon slope, however, was appreciably greater (9.18 $\mathrm{m} \mathrm{min}^{-1}$ decade).

For women, there were also no significant differences in the slopes among the different events up to the $1,500 \mathrm{~m}$ ( $b$ in the figure). The slope, however, was approximately double that for the men, ranging from 14.04 to $17.86 \mathrm{~m} \mathrm{~min}^{-1}$ decade. As for men, the rate at which $\bar{V}$ increased in the marathon was appreciably greater (37.75 m min $\mathrm{m}^{-1}$ decade). Despite the potential pitfalls, we could not resist extrapolating these record progressions into the future.

Unless the progression rate of mens' records increases relative to that of women, then $\bar{V}$ for these events will be

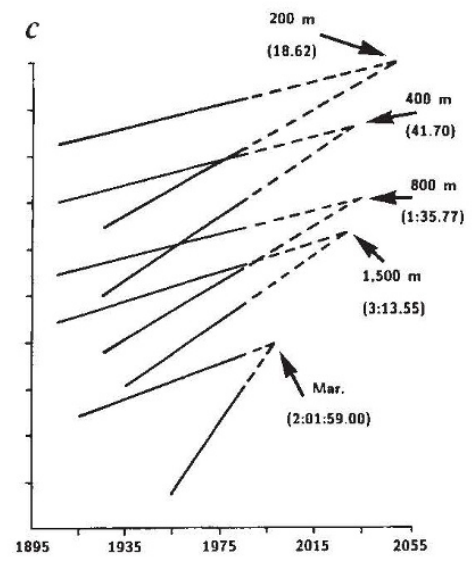

no different for men and women within the first half of the twenty-first century ( $c$ in the figure). Beyond that time, current progression rates imply superior performance by women. The projected intersection for the marathon is 1998 .

The suggestion that women could, so soon, be running these races as fast as men seems improbable at first appearance. None of the current women's world record holders at these events could even meet the men's qualifying

standard to compete in the 1992 Olympic games. However, it is the rates of improvement that are so strikingly different - the gap is progressively closing6.

Although it is difficult to establish a precise metabolic energy equivalent of these rates of improvement, one may estimate from the data of Margaria $e t$ al. ${ }^{7}$ and Wyndham et al. ${ }^{8}$ that, for the events up to $10,000 \mathrm{~m}$, the progression requires a rate of increase in $\mathrm{O}_{2}$ consumption of about $10 \mathrm{ml} \mathrm{min}^{-1} \mathrm{yr}$ for men and more than double that for women.

It is unlikely that we will learn when, and how rapidly, the current high rate of improvement was established, owing to the lack of reliable times over reliable distances in the past. Some world records are available however, as far back as the 1860 s (ref. 4); they are consistent with the values 'expected' from the current progression rates. Whether the world record progression rate will begin to slow, either relatively abruptly or more progressively, will only become apparent in the future.

In any event these results pose four challenging questions to physiologists. Why is: the world record progression in the various events so linear over an interval of approximately a century; the slope of the record progression so similar from the sprints to the $10,000 \mathrm{~m}$; the record progression in the marathon appreciably greater; and the record progressions for women increasing at such a rapid rate relative to men?

BRIAN J. WHIPP

SUSAN A. WARD

Departments of Physiology and

Anesthesiology,

School of Medicine,

University of California,

Los Angeles, California 90024, USA

1. Lloyd, B. B. Circ. Res. $\mathbf{X X} \& \mathbf{X X \mathbf { X }}$ (suppl. 1), |218-226 (1967).

2. Di Prampero, P. E. Rivista di Cultura Sportiva 3, 3-7 (1984).

3. Matthews, P. Track and Field Athletics: The Records (Guinness, Enfield, 1986)

4. Progression of World's Best Performances and Official I.A.A.F. World Records (International Athletic Foundation, Monaco, 1987).

5. Ryder, H. W., Carr, H. J. \& Herget, P. Sci. Am. 234, 6 109-119 (1976)

6. Dyer, K. J. J. Biosocial Sci. 9, 325-338 (1977)

7. Margaria, R. Cerretelli, P., Aghemo, P. \& Sassi, G. J. appl. Physiol. 18, 367-371, (1963).

8. Wyndham, C. H., Strydom, N. B., Van Rensburg, A. J. \& Benade, A. J. S. S. Afr. Med. J. 43, 996-1002 (1969).

\section{How predictable is chaos?}

SIR - Sugihara and May ${ }^{1}$ have developed a novel approach to distinguish chaos from noise in short time series. The principle lies in a geometric method designed to make predictions based on a library of past patterns in the series, with a view of comparing them with actual trajectories. The necessity for such a technique arises in fields like population biology, where data are typically sparse. Without questioning the basis of the approach, we point out that limitations exist: the method fails to detect chaos in series of data simulated by a simple and general age-structured population model.

One reason is that corresponding strange attractors are split into several disconnected pieces. The iterations of any point chosen in one of the fragments are located successively in the other pieces (in a precise order), before falling back into the initial one, and so on. As a consequence, there is an overall period in the structure of the attractors, which is equal to the number of pieces. It has been known for some time that this pattern occurs in discrete systems when the transition towards chaos runs through an invariant circle that is kinked $^{2}$. Such bifurcations are common in ecological models (see refs 3 and 4). They compromise attempts to identify chaotic dynamics with the method of Sugihara and May, which is influenced by the overall periodicity of the attractors, whereas chaos is hidden in what may well be classified as 'residual noise'. More surprisingly, this difficulty still per- 